\title{
Stochastic performances estimate of a universal and flexible power management system for the future European electricity network
}

\author{
Micaela Caserza Magro, Stefano Savio \\ Department of Electrical Engineering - University of Genova \\ Via Opera Pia 11a - 16145 Genova - Italy \\ Tel. +390103532182 - e-mail: caserza@die.unige.it, stefano.savio@unige.it
}

\begin{abstract}
This paper presents the final results of the activities carried out by the authors to estimate the availability performances of an innovative modular power conversion architecture, developed within the UNIFLEX-PM (Universal and Flexible Power Management) EU project. The performed study also addresses the analysis of the effects on system stochastic performances deriving from basic components wear out phenomena, and the ones deriving from the adoption of different redundancy strategies and maintenance policies. The UNIFLEX-PM architecture represents an innovative solution for power management in the distribution networks, thus the reliability and availability studies have been focused on obtaining basic figures useful to define the effectiveness of the proposed solution through comparison with a power converter architecture already used for building commercial products and assumed as the reference case.
\end{abstract}

\section{Keywords}

Power conversion, power distribution, reliability, availability, Monte Carlo method.

\section{Introduction}

The electricity network is evolving and changing its structure. We are all observing an evolution towards what it is called the Future European Electricity Network [1]. The challenge for creating a new network comes from the highlight defined in 2001 by the Green paper "Towards a European strategy for the security of energy supply” in which the European Commission stressed the weaknesses of the European economy regarding energy dependence. For solving this problem it is necessary to use and exploit the potentialities of sustainable resources. In such a scenario the UNIFLEX-PM project application finds its origin [2]. The project is a European Project of the $6^{\text {th }}$ Framework Programme co-financed in the sustainable energy systems call, and it proposes an innovative modular power converter architecture that can satisfy the requirements of Future European Electricity Network. The innovative architecture is based on Medium Frequency (MF) transformer as isolation stage and on IGBT modules as static switches.

Reliability and availability studies have been carried out by the authors to identify the main stochastic performances of the application and evaluate its effectiveness. To this aim, the reliability and availability measures have been compared with the ones performed on an architecture already existing, used for developing commercial products and characterised by functionalities similar to the UNIFLEX-PM ones.

As far as the paper contents are concerned, the first section deals with a brief description of the UNIFLEX$\mathrm{PM}$ and reference case architectures. Once identified the basic components and their reliability characteristics, the general assumptions taken into account for the dependability analysis for both UNIFLEX-PM and the reference case are then presented. The last section of the paper deals with the availability estimate process, carried out through a dedicated software tool developed by the authors and based on the Monte Carlo method: the availability results for UNIFLEX-PM and for the reference case are reported and discussed.

\section{UNIFLEX-PM system}

The UNIFLEX-PM system is characterised by a threephase architecture, adopting multi-level converters (IGBT technology based), which allows a bidirectional power flow.

The proposed solution is based on a DC/DC isolation stage $[3,4]$, characterized by a symmetrical configuration: there are four conversion stages, two by two equal. In particular, as depicted in Fig.1, the following macroblocks are present in each level:

- an input macro block consisting of AC/DC and DC/AC conversion units with interposed a DC link;

- a Medium Frequency (MF) transformer for isolation purposes;

- an output macro block consisting once again of $\mathrm{AC} / \mathrm{DC}$ and DC/AC conversion units with interposed a DC link.

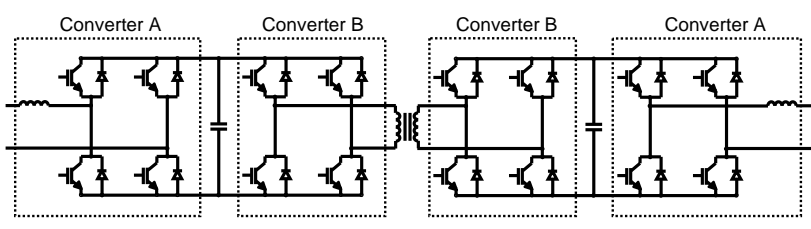

Fig. 1. UNIFLEX-PM architecture (DC/DC isolation stage) one level 
UNIFLEX-PM may be conceived as a power converter having three ports, as the schematic overview presented in Fig. 2 shows. In such an example, taken into account by the Consortium for building the prototype and by the authors in the paper for the reliability studies, two ports are directly connected to the $3.3 \mathrm{kV}$ distribution network and the third port is connected to the $415 \mathrm{~V}$ busbars, where Renewable Energy Systems (RES) and storage elements are connected. The power handled by the threephase module has been supposed equal to $300 \mathrm{kVA}$ and for each phase four levels are foreseen.

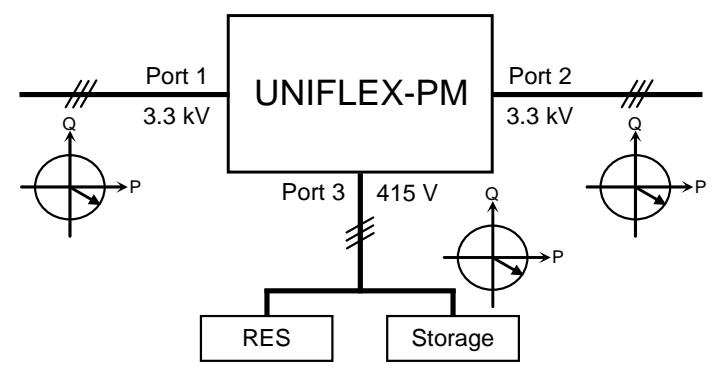

Fig. 2. Schematic overview of the UNIFLEX-PM application (example)

For the reliability estimate purposes, the system has been decomposed into basic assemblies/items, and the number of assemblies needed to build the whole application computed as reported in Table I.

Table I. - Assemblies for the 3-phase UNIFLEX-PM architecture

\begin{tabular}{|l|c|}
\hline \multicolumn{1}{|c|}{ Assembly } & No. of elements \\
\hline Input filter & 12 \\
\hline Converter AC/DC & 24 \\
\hline DC link & 24 \\
\hline Converter DC/AC & 24 \\
\hline MF transformer & 12 \\
\hline Output filter & 12 \\
\hline Transducers system & 1 \\
\hline Control & 1 \\
\hline
\end{tabular}

\section{Reference case: Modular Multilevel Converter}

For performing the impact analysis it has been judged necessary to identify a suitable reference case, represented by an existing solution with analogous functionalities, in order to evaluate the impacts (possible benefits) deriving from the adoption of the UNIFLEXPM solution by comparison. As described in the following, the assumed reference case is an architecture based on Modular Multilevel Converters (usually identified with the acronym $\mathrm{M}^{2} \mathrm{LC}$ ), that is today adopted for industrial products and that provides a subset of the functionalities characterizing the UNIFLEX-PM system.

The selected reference case is a High Voltage Direct Current (HVDC) converter, having a four quadrant operation capability, based on the Modular Multilevel Converter $\left(\mathrm{M}^{2} \mathrm{LC}\right)$ architecture. The converter has a modular multilevel conversion structure, with IGBTs as static switch. It is possible to have different connection schemes for the HVDC solution, but the interesting one for the impact analysis purposes is the so called back-toback configuration. In this case the rectifier and the inverter are located in the same station. Several international companies have used the $\mathrm{M}^{2} \mathrm{LC}$ architecture for developing commercial HVDC products in the backto-back configuration. Considering this architecture, the transformer connecting the converter stage and the external AC grid is a normal two winding distribution transformer.

With reference to Fig. 3 and Fig. 4, this topology has at least two peculiar features:

- the whole converter consists of a chosen number of identical Sub Modules (SM);

- any additional central components should be avoided, as the DC link capacitors which are distributed inside the single Sub Module.

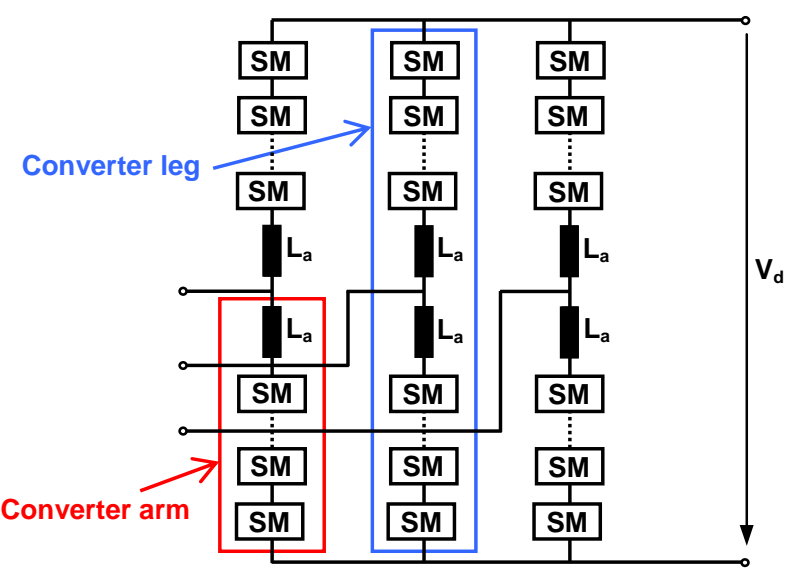

Fig. 3. Modular Multilevel Converter architecture

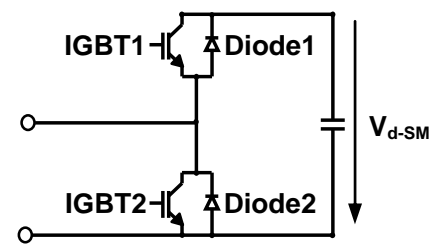

Fig. 4. Sub Module architecture

A converter arm, which consists of $n$ Sub Modules, represents a controllable voltage source. Essentially, the topology reported in Fig. 3 represents a Voltage Source Converter (VSC), which is able to control the AC voltages (multilevel) and the DC-bus-voltage $\left(\mathrm{V}_{\mathrm{d}}\right)$ via the switching states of the Sub Modules.

Taking into account the information already provided, the following considerations apply:

- AC/DC and DC/AC conversion stages are built with two Modular Multilevel Converters;

- each converter may be decomposed into three phase units;

- $\quad$ each phase unit is built with $2 * n$ Sub Modules;

- each Sub Module is built with a bidirectional switch (two IGBTs and two diodes) and a power capacitor, which represents a part of the DC link, as intended in the old VSC architectures. 
A rough design of the $M^{2} L C$ architecture for an application having a size comparable to UNIFLEX-PM has been defined according to $[5,6,7,8]$. Aim of such preliminary design is to identify number and rating of the basic components of the converter to be utilized during the reliability prediction study. A $300 \mathrm{kVA}$ at $3.3 \mathrm{kV}$ $\mathrm{M}^{2} \mathrm{LC}$ based HVDC application requires five levels per arm (this means ten Sub Modules per converter leg and phase) for both the AC/DC conversion stage and the $\mathrm{DC} / \mathrm{AC}$ conversion stage. Also for the reference case the number of assemblies has been computed, as shown in Table II.

Table II. - Assemblies for the M $^{2}$ LC based HVDC application

\begin{tabular}{|l|c|}
\hline \multicolumn{1}{|c|}{ Assembly } & No. of elements \\
\hline Input filter & 3 \\
\hline AC/DC converter (3 legs) & 1 \\
\hline DC/AC converter (3 legs) & 1 \\
\hline Isolation transformer & 2 \\
\hline Output filter & 3 \\
\hline Transducers system & 1 \\
\hline Control equipment & 1 \\
\hline
\end{tabular}

\section{Components reliability and general assumptions}

Usually, reliability studies for systems where just electrical and electronic components are present are carried on considering only the useful life of all the components (Time To Failure exponentially distributed). If the prediction deals with a system characterized, like UNIFLEX-PM, by a long life cycle (more than 10 years), it is reasonable that some of the items start experiencing wear-out effects. Thus, such phenomenon should be duly taken into account for the reliability estimate. Considering wear-out effects means that for the affected components the hazard rate is not constant but it starts to increase with time. Wear-out impact at the system hierarchical level has been investigated for the following three components:

- the capacitor (electrolytic for UNIFLEX-PM and film based for the reference case);

- the transformer (MF for UNIFLEX-PM and normal distribution transformer for the reference case);

- the IGBT module.

In particular, the reliability behaviour of the components affected by wear-out has been modelled with two different distributions: an exponential distribution with a constant failure rate (1) plus a Weibull distribution to take into account wear-out occurrence, as described in (2).

$$
\begin{gathered}
R(t)=e^{-\lambda t} \\
R(t)= \begin{cases}e^{-\left(\frac{t-\gamma}{\eta}\right)^{\beta}} & t \geq \gamma \\
0 & t<\gamma\end{cases}
\end{gathered}
$$

As far as the symbols utilised in the previous two relationships are concerned:
- $R(t)$ is the reliability at time $t$;

- $\lambda$ is the constant hazard rate (failure rate) for the item;

- $\beta$ is the shape factor (if $\beta>1$ the hazard rate increases);

- $\quad \eta$ is the scale parameter, or characteristic life, it is the life at which $63.2 \%$ of the population has failed;

- $\quad \gamma$ is the failure free time, namely the starting time of the distribution.

Table III summarizes the values of the parameters used for modelling the components affected by wear-out. Those values have been identified taking into account, for the capacitors and the isolation transformer, the extensive literature existing about, and, for the MF transformer and the IGBT module, the information provided by the manufactures within the Consortium.

Table III. - Parameters of the Weibull distributions (wear-out)

\begin{tabular}{|l|c|c|c|}
\hline \multicolumn{1}{|c|}{ Component } & $\boldsymbol{\beta}$ & $\boldsymbol{\eta}$ [h] & $\boldsymbol{\gamma}$ [h] \\
\hline Electrolytic capacitor & 3 & 15000 & 40000 \\
\hline Film capacitor & 3 & 30000 & 100000 \\
\hline MF Transformer & 3 & 50000 & 80000 \\
\hline Isolation transformer & 3 & 50000 & 80000 \\
\hline IGBT module & 3 & 50000 & 80000 \\
\hline
\end{tabular}

When wear-out effects have to be estimated, the reliability prediction is usually a not trivial task; for such reasons it was decided to perform the analysis by means of a dedicated commercial SW tool, namely BlockSim ${ }^{\circledR}$ v.7 by ReliaSoft.

The reliability prediction has been performed in three different working conditions:

- components in rated operating conditions (base reliability): the failure rate of each component of the system is referred to the rated conditions of the component itself;

- system in rated operating conditions: the failure rate of each component of the system is referred to the rated operating conditions of the system, which means the grid voltage equals $3.3 \mathrm{kV}$ and the apparent power $300 \mathrm{kVA}$;

- system in real operating conditions: the failure rate of each component of the system is referred to the real operating conditions of the system, which have been identified by means of a load profile considering the AC grid always at $3.3 \mathrm{kV}$. The definition of the load profile refers to power consumption data recorded for a period of seven months in a village of Jutland, Denmark. The recorded data may be assumed as representative of a possible real operating scenario for the UNIFLEX-PM system.

For all the basic elements of the UNIFLEX-PM system, the adopted values of the failure rate $\lambda$ are reported in Table IV ( 1 FIT equals $10^{-9}$ failures/h). Base values are derived from $[9,10,11]$ for all the components but IGBT modules and MF transformers, whose failure rate value, not covered in literature, has been directly provided by manufacturers. Failure rate values for the other working conditions have been computed by means of suitable 
models, based on a procedure analogous to the stress analysis in [12], where applicable. On the contrary, it has been assumed the working conditions do not affect the parameters of the Weibull distribution for the components that experience wear-out. At last, current and voltage transducers have not been taken into account due to the negligible value of their failure rate, and the same applies to the DC link resistors, whose failure rate is negligible if compared with the one of the other basic components of the macro-block they belong to.

Table IV. - Components failure rate (UNIFLEX-PM)

\begin{tabular}{|l|c|c|c|}
\hline \multicolumn{1}{|c|}{ Item } & $\boldsymbol{\lambda}_{\text {base }}$ [FIT] & $\boldsymbol{\lambda}_{\text {rated }}$ [FIT] & $\boldsymbol{\lambda}_{\text {real }}$ [FIT] \\
\hline Inductor & 80 & 80 & 80 \\
\hline Resistor & 12 & 12 & 12 \\
\hline IGBT switch & 25 & 15 & 12 \\
\hline Gate driver & 25 & 25 & 25 \\
\hline Capacitor (electrolytic) & 40 & 40 & 40 \\
\hline MF transformer & 900 & 900 & 513 \\
\hline FPGA & 5800 & 5800 & 5800 \\
\hline DSP & 110 & 110 & 110 \\
\hline
\end{tabular}

Table V reports the failure rate of the basic components of the reference case. As for the UNIFLEX-PM system some items, namely the current and voltage transducers, have not been taken into account.

Table V. - Components failure rate ( $\mathrm{M}^{2} \mathrm{LC}$ based HVDC system)

\begin{tabular}{|l|c|c|c|}
\hline \multicolumn{1}{|c|}{ Item } & $\boldsymbol{\lambda}_{\text {base }}$ [FIT] & $\boldsymbol{\lambda}_{\text {rated }}$ [FIT] & $\boldsymbol{\lambda}_{\text {real }}$ [FIT] \\
\hline Inductor choke & 80 & 80 & 80 \\
\hline Resistor & 12 & 12 & 12 \\
\hline IGBT switch & 25 & 15 & 12 \\
\hline Gate driver & 25 & 25 & 25 \\
\hline Capacitor (PPG film) & 300 & 300 & 300 \\
\hline Isolation transformer & 1600 & 1600 & 910 \\
\hline FPGA & 5800 & 5800 & 5800 \\
\hline DSP & 110 & 110 & 110 \\
\hline
\end{tabular}

For UNIFLEX-PM the dependability study considered also possible solutions for improving the performances of the application; in particular, the impact deriving from the implementation of redundancy strategies has been investigated. The following two architectures have been considered:

- one redundant (stand-by) level for each phase: each phase has five levels (four active + one stand-by) and the successful completion of the mission requires that four levels are correctly operating (4-out-of-5 redundancy configuration through a stand-by unit);

- one redundant (stand-by) level for the complete threephase system: each phase has four operating levels and if one level of a generic phase is down, it is possible to switch on the stand-by level (12-out-of-13 redundancy configuration through a stand-by unit).

Moreover, the preliminary analysis performed showed that the bottleneck from the reliability point of view was represented for UNIFLEX-PM by the electrolytic capacitors used for the DC link. For such a reason, the possible benefits deriving from the adoption of another family of capacitors, namely the polypropylene film capacitors used for the reference case, was also investigated.

\section{Availability modelling approach}

Instantaneous availability $A(t)$ is a function of time and is defined as the probability that a system is ready to correctly perform its functions at time $t$, under specific working conditions. Respect to the reliability, which is a function of a certain time interval, the availability is measured at a certain time. The availability is often chosen as a specific requirement for a repairable system, whose target is to provide the required functionalities in a continuative way. The availability of a repairable system is usually a function of the failure rate $\lambda$ and the repair rate $\mu$, both constant supposing Time To Failure (TTF) and Time To Repair (TTR) exponentially distributed. For a simple unit, with a constant failure rate $\lambda$ and a constant repair rate $\mu$, the instantaneous availability and its steadystate value $A_{S S}$ can be computed as it follows:

$$
\begin{gathered}
A(t)=\frac{\mu}{\lambda+\mu}+\frac{\lambda}{\lambda+\mu} e^{-(\lambda+\mu) t} \\
A_{S S}=\lim _{t \rightarrow \infty} A(t)=\frac{\mu}{\lambda+\mu}=\frac{M T T F}{M T T F+M T T R}
\end{gathered}
$$

where the Mean Time To Failure (MTTF) and the Mean Time To Repair (MTTR) are equal to $1 / \lambda$ and $1 / \mu$ respectively.

Thus the asymptotic (or steady-state) availability may be computed as the ratio between the time the system has been correctly operating and the calendar time (for large values of the latter, to be quantified according to the dependability parameters). The authors of [13] have demonstrated that this consideration also applies (under general conditions) for failure rates $\lambda(t)$ and repair rates $\mu(t)$ which are not constant.

This means that also considering a non exponential distribution for the TTF and the TTR of the system components, the steady state availability of the entire system can be expressed as the ratio between the time the system has been working and the total calendar time.

Different approaches may be utilised for carrying out the availability estimate of a system, such as the simple combinatorial methods or the more complex Markov Chains or Stochastic Petri Nets, being the major constraint usually represented by the possibility to just analyse system where failure and repair times are exponentially distributed. As a consequence those methods cannot be successfully utilised for the UNIFLEX-PM availability estimate, where some items are characterised by wear-out (and the relevant TTF not exponentially distributed). To overcome the problem authors have decided to perform the analysis by means of the Monte Carlo approach, a very powerful simulation based method, which allows to estimate the dependability performances of a system thanks to a hybrid method, by merging a "deterministic" analysis of the behaviour of a 
sample of the population and a statistical post-processing of the results.

The Monte Carlo method is utilised for the generation of the Times To Failure and of the Times To Repair of the different components over a predefined time horizon, once known their reliability and maintainability characteristics. The Time To Failure and the Time To Repair are random variables characterised by their relevant Probability Density Functions (PDFs). The knowledge of such PDFs is mandatory for performing the reliability and availability analysis of the system, as the Cumulative Distribution Function (CDF) $F(t)$ of a random variable can be computed starting from the relevant $\operatorname{PDF} f(t)$ as it follows:

$$
F\left(t_{0}\right)=\int_{0}^{t_{0}} f(t) d t
$$

being $F\left(t_{0}\right)$ the probability that the stochastic variable $t$ is not greater than a given value $t_{0}$, and $t \in(0,+\infty)$. For each value of the random variable $t$ in $(0,+\infty), F(t)$ assumes values uniformly distributed in $(0,1)$. So, elements of a sample of the random variable $t$ distributed according to $F(t)$ can be obtained generating a random number in $(0,1)$ and subsequently inverting $F(t)$. For instance, if the random variable $t$ is exponentially distributed, the Cumulative Distribution Function $F(t)$ may be expressed as it follows, where $\lambda$ is a constant:

$$
F(t)=1-e^{-\lambda t}
$$

If the TTF of a component is characterised by such a distribution, one element of a generic sample can be generated solving the following equation:

$$
\mathrm{TTF}=\frac{\ln (1-F)}{-\lambda}
$$

where $\lambda$ is the reciprocal of the component MTTF and $F$ is, for any element of the sample to be generated, a random number in $(0,1)$ get by Monte Carlo method. The Monte Carlo based is usually due to its flexibility, as it allows to carry out the failure analysis for components whose TTF (or TTR) is characterised by any distribution. According to the reliability connection of the basic components of the system (series, parallel, stand-by, $k$ out-of- $n, \ldots$ ) and the level of detail chosen for modelling its real structure, it is possible to analyse the behaviour of the system into a state space, where transitions from one state to another are driven by the TTF (or TTR) values get by Monte Carlo method. The result of such an analysis just represents one element of the sample of the population. Once collected a suitable number of elements, a statistical post-processing is then carried out to estimate the desired stochastic measures.

To carry out the availability estimate for both UNIFLEX$\mathrm{PM}$ and the reference case, a software tool has been developed using Matlab 7.0 environment. The tool implements the Monte Carlo method for the two architectures and the interface has been designed to allow the user to friendly insert all the reliability, maintainability and simulation parameters needed for performing the analysis, taking also into account components characterised by TTF and TTR not exponentially distributed.

\section{Availability prediction results}

As previously mentioned, in order to make a fair comparison between the two solutions, simulations have been carried out taking into account three different working conditions (base, rated and real). Moreover, as far as UNIFLEX-PM is concerned, the impact deriving from the adoption of different architectures (basic - no redundancy strategies, 4-out-of-5 redundancy and 12-outof-13 redundancy) and different technologies for DC link capacitors (aluminium electrolytic and polypropylene film) has been investigated.

Table VI reports for each basic item its MTTR, that represents the mean time required to have the spare unit at the application site (supposing it is available at the maintenance depot), perform troubleshooting process, replace the faulted item and restart the system. It is worth reminding that TTR has been considered exponentially distributed and a single repair policy has been assumed.

Simulation results are reported in Table VII; each simulation covers a time interval of 90000 hours and for each case study 10000 simulations have been performed. For each operating scenario the mean availability and the Mean Time To First Failure (MTTFF), together with the relevant Standard Deviation (SD), are shown; the mean number of system failures occurred is reported as well. As Table VII clearly shows, the performances of the UNIFLEX-PM system (base architecture, no redundancy) and of the $\mathrm{M}^{2} \mathrm{LC}$ solution are comparable in terms of availability and MTTFF.

Table VI. - Components MTTR

\begin{tabular}{|l|c|}
\hline \multicolumn{1}{|c|}{ Component } & MTTR [h] \\
\hline IGBT module & 25 \\
\hline DC link & 25 \\
\hline MF transformer & 80 \\
\hline Isolation transformer & 100 \\
\hline Input/output filter & 20 \\
\hline Control & 40 \\
\hline
\end{tabular}

The dependability characteristics of the $M^{2} L C$ solution have however a better behaviour. In particular, stated that the MTTFF of the reference case is higher of about 15$20 \%$, the number of its failures is dramatically lower (about $85-90 \%$ ), if compared with the UNIFLEX-PM system.

This huge difference in number of failures is due to the different technology used for the DC link capacitors. The electrolytic capacitors, used in the design of the UNIFLEX-PM prototype, are characterised by an early start of the wear-out phenomena, if compared with the time horizon considered. 
TABLE VII. - Summary of the dependability performances

\begin{tabular}{|c|c|c|c|c|c|}
\hline & \multicolumn{2}{|c|}{ Mean Availability } & \multicolumn{2}{|c|}{ MTTFF } & \multirow{2}{*}{ Failures } \\
\hline & Value [\%] & SD [\%] & Value [h] & SD [h] & \\
\hline \multicolumn{6}{|c|}{ UNIFLEX-PM (electrolytic capacitors) - base working conditions } \\
\hline No redundancy & 99.1 & $2 e-5$ & 22342 & 151 & 29.4 \\
\hline 4-out-of-5 redundancy & 99.8 & $1 \mathrm{e}-5$ & 35574 & 127 & 8.2 \\
\hline 12-out-of-13 redundancy & 99.7 & $1 \mathrm{e}-5$ & 32712 & 132 & 8.3 \\
\hline \multicolumn{6}{|c|}{ UNIFLEX-PM (electrolytic capacitors) - rated working conditions } \\
\hline No redundancy & 99.1 & $2 \mathrm{e}-5$ & 23209 & 152 & 29.2 \\
\hline 4-out-of-5 redundancy & 99.8 & 1e-5 & 35986 & 125 & 8.1 \\
\hline 12-out-of-13 redundancy & 99.7 & $1 \mathrm{e}-5$ & 33120 & 130 & 8.2 \\
\hline \multicolumn{6}{|c|}{ UNIFLEX-PM (electrolytic capacitors) - real working conditions } \\
\hline No redundancy & 99.1 & $2 \mathrm{e}-5$ & 25190 & 153 & 28.8 \\
\hline 4-out-of-5 redundancy & 99.8 & $1 \mathrm{e}-5$ & 36814 & 122 & 8.0 \\
\hline 12-out-of-13 redundancy & 99.8 & $1 \mathrm{e}-5$ & 34572 & 127 & 8.1 \\
\hline \multicolumn{6}{|c|}{ UNIFLEX-PM (film capacitors) - base working conditions } \\
\hline No redundancy & 99.8 & $2 \mathrm{e}-5$ & 26786 & 241 & 4.0 \\
\hline 4-out-of-5 redundancy & 99.9 & $7 \mathrm{e}-6$ & 55879 & 306 & 1.3 \\
\hline 12-out-of-13 redundancy & 99.9 & $9 e-6$ & 46459 & 284 & 1.6 \\
\hline \multicolumn{6}{|c|}{ UNIFLEX-PM (film capacitors) - rated working conditions } \\
\hline No redundancy & 99.8 & $2 e-5$ & 27769 & 247 & 3.9 \\
\hline 4-out-of-5 redundancy & 99.9 & $7 e-6$ & 57263 & 307 & 1.3 \\
\hline 12-out-of-13 redundancy & 99.9 & $9 e-6$ & 47532 & 292 & 1.6 \\
\hline \multicolumn{6}{|c|}{ UNIFLEX-PM (film capacitors) - real working conditions } \\
\hline No redundancy & 99.8 & $1 \mathrm{e}-5$ & 31802 & 269 & 3.4 \\
\hline 4-out-of-5 redundancy & 99.9 & $6 e-6$ & 60415 & 309 & 1.2 \\
\hline 12-out-of-13 redundancy & 99.9 & $8 \mathrm{e}-6$ & 51611 & 301 & 1.5 \\
\hline \multicolumn{6}{|c|}{$\mathrm{M}^{2} \mathrm{LC}$ based HVDC system } \\
\hline Base & 99.8 & $2 e-5$ & 28027 & 248 & 3.5 \\
\hline Rated conditions & 99.8 & $2 e-5$ & 28796 & 253 & 3.4 \\
\hline Real working conditions & 99.8 & $2 \mathrm{e}-5$ & 29426 & 256 & 3.4 \\
\hline
\end{tabular}

This means a high number of capacitor failures, whose effect is mitigated, as far as availability is concerned, by the small value (compared with the total simulated time) of the Mean Time To Repair required to carry out the corrective maintenance on the DC link equipment. This fact allows to achieve very good availability figures for the UNIFLEX-PM system too.

As far as the availability is concerned, Table VII shows that the difference between the base UNIFLEX-PM architecture and the reference case is less than $1 \%$.

Starting from the encouraging results of the first analysis, which has testified UNIFLEX-PM (base architecture) and the reference case are comparable from the dependability point of view, some strategies for increasing the availability of the UNIFLEX-PM system and decreasing the total number of system failures have been investigated (decreasing the total number of system failures means reducing management and operating costs).

From the results dealing with real operating conditions, it turns out that considering the 4-out-of-5 redundancy the UNIFLEX-PM availability increases from $99.1 \%$ to $99.8 \%$, while the number of system failures decreases from around 29 to 8 . This redundancy strategy implies also an increase of the MTTFF of about 45\%. This solution, however, has a drawback, which is the cost of implementing four additional levels and to customise the control software to switch from the failed level to the stand-by one.
This is the reason for the additional analysis carried out and dealing with the 12-out-of-13 redundancy strategy, where just one stand-by level for the entire UNIFLEXPM application is foreseen. Such solution presents a less impact in terms of costs, stated that the problem of the implementation of the switching strategies is still present.

The results testify that the availability performances of this solution is still good and comparable with the 4-outof-5 redundancy strategy: availability is around $99.8 \%$ and the number of system failures is about 8 for the whole time interval. The MTTFF decreases in comparison to the 4-out-of-5 configuration, but it is still higher than the one of the base architecture. In particular, the MTTFF decreases of about $6 \%$ with reference to the 4-out-of-5 redundancy strategy, but increases of about $35 \%$ with reference to the base architecture.

The detailed analysis of the system availability for UNIFLEX-PM (base architecture, no redundancy) shows that the component which mostly impacts on such stochastic parameter is the DC link capacitor, due to the early start of the wear-out process. Thus, an increase of the availability and a reduction of the system failures could be achieved changing the technology of the DC link capacitors, so that the wear-out phenomena can be suitably postponed.

With a different (film based) technology for the DC link capacitors it is possible to reach availability performances comparable with the ones obtained using some redundancy strategies; in particular the mean 
availability can reach $99.8 \%$, while the number of failures is reduced to about 4 and the MTTFF shows an increase of about $20 \%$ or more respect to the base architectural solution.

\section{Preventive maintenance on capacitors}

As Table VII clearly shows, the electrolytic capacitors in the basic UNIFLEX-PM solution are, from the dependability point of view, one of the most delicate elements due to the early start of the wear-out phenomena.

Thus, a possible solution for mitigating the impact of the wear-out on the stochastic performances of the system is to adopt a suitable preventive maintenance policy for such items. In such a context, the implementation of a preventive maintenance procedure means the identification of a suitable time interval after which all the capacitors of the DC links are replaced, in order to avoid the occurrence of failures due to wear-out. Of course the time interval after which the capacitors are replaced should be similar to the time at which the wearout of the component starts, in order to maximise the benefits of the procedure (or to avoid, sometimes, the preventive maintenance action negatively impacts on the overall availability performances of the system). The preventive maintenance action is completely identified inside the simulator by the following two parameters:

- the time interval after which the components are replaced;

- the MTTR needed for a DC link replacement.

As far as the second parameter is concerned, it is worth mentioning that the MTTR needed to substitute each DC link during a preventive action can be assumed lower than the time necessary to perform a corrective repair action after a fault on the same equipment, because the term "preventive" means the maintenance actions have been suitably planned. A first set of simulations was carried out to estimate the impact of the DC link replacing interval on the dependability performances of the UNIFLEX-PM system (rated operating conditions). The MTTR values considered for the basic components of the system are the ones already described in Table VI, while one hour is the MTTR assumed for completing a preventive maintenance action on each single DC link. At last, the simulated time horizon equals 90000 hours.

The results are reported in Table VIII and show that preventive maintenance actions produce a sensible reduction of the total number of system failures if they are performed with a frequency not higher than $40000 \mathrm{~h}$ (from about 29, when just corrective maintenance actions are implemented, to about 4). This is mainly due to the fact failures related to capacitor wear-out are avoided, and just the random failures of the basic components do exist. It is worth mentioning that wear-out phenomena for the other components (MF transformer and IGBT modules) starts very close to the end of the simulation period and their impact has a not negligible weight in the long term availability only (more than 90000 hours).
Table VIII. - Sensitivity analysis on preventive maintenance intervals (DC link MTTR = $1 \mathrm{~h}$ )

\begin{tabular}{|c|c|c|c|c|c|}
\hline $\begin{array}{c}\text { Time } \\
\text { interval } \\
\text { [h] }\end{array}$ & \multicolumn{2}{|c|}{ Mean Availability } & \multicolumn{2}{c|}{ MTTFF } & Failures \\
\hline 20000 & 99.7 & 2e-5 & 28757 & 252 & 3.8 \\
\hline 25000 & 99.7 & $2 \mathrm{e}-5$ & 28820 & 251 & 3.7 \\
\hline 30000 & 99.7 & $1 \mathrm{e}-5$ & 29144 & 253 & 3.7 \\
\hline 35000 & 99.7 & 2e-5 & 29310 & 255 & 3.6 \\
\hline 40000 & 99.7 & 2e-5 & 28991 & 252 & 3.8 \\
\hline 45000 & 99.5 & 2e-5 & 23219 & 153 & 12.2 \\
\hline 50000 & 99.3 & 2e-5 & 23001 & 153 & 22.2 \\
\hline 55000 & 99.1 & 2e-5 & 22846 & 152 & 27.2 \\
\hline 60000 & 99.1 & 2e-5 & 22984 & 151 & 27.4 \\
\hline
\end{tabular}

Moreover, the results show that preventive maintenance actions carried out before the starting time of the wearout phenomena for the DC link capacitors (40000 hours), cause an increase of the mean availability of more than $0.5 \%$, if compared with the value of such quantity in the same working conditions (rated conditions) and corrective maintenance only.

If the preventive actions are carried out behind 40000 hours, the mentioned benefits, in terms of both number of failures and availability figures, are smoothed and the stochastic performances of the system tend to get similar to the ones presented in Table VII.

Additional simulations were carried out for a higher value of the MTTR needed to complete the preventive replacement of each single DC link (five hours). The results are presented in Table IX and are very interesting because they show the consequences related to the speed of the maintenance actions.

In particular, if the preventive action on the DC link is not fast enough, the positive effect of the preventive replacement on the availability performances of the system is smoothed. On the contrary, Table IX shows that if the preventive action is done before the starting of wear-out, the decrease of the number of the system failures is always really significant (while such effect is once again reduced if the maintenance interval increases).

Table IX. - Sensitivity analysis on preventive maintenance intervals (DC link MTTR $=5 \mathrm{~h}$ )

\begin{tabular}{|c|c|c|c|c|c|}
\hline $\begin{array}{c}\text { Time } \\
\text { interval } \\
\text { [h] }\end{array}$ & \multicolumn{2}{|c|}{ Mean Availability } & \multicolumn{2}{c|}{ MTTFF } & Value [\%] \\
VD [\%] & Value [h] & SD [h] & Failures \\
\hline 20000 & 99.3 & 2e-5 & 28317 & 253 & 3.6 \\
\hline 25000 & 99.4 & 2e-5 & 28602 & 251 & 3.7 \\
\hline 30000 & 99.4 & 2e-5 & 29008 & 250 & 3.7 \\
\hline 35000 & 99.5 & 2e-5 & 29264 & 255 & 3.7 \\
\hline 40000 & 99.5 & 2e-5 & 28872 & 251 & 3.7 \\
\hline 45000 & 99.3 & 2e-5 & 22907 & 151 & 12.0 \\
\hline 50000 & 99.2 & 2e-5 & 23187 & 152 & 22.1 \\
\hline 55000 & 99.0 & 2e-5 & 22924 & 152 & 27.2 \\
\hline 60000 & 99.0 & 2e-5 & 22935 & 152 & 27.4 \\
\hline
\end{tabular}

It is worth mentioning that the MTTFF value presented in Table VIII and Table IX refers to the calendar time before the first system failure occurs and it may include the preventive maintenance time. 


\section{Conclusions}

The paper has presented the final results of the activities carried out by the authors to estimate the effectiveness of an innovative power management system, namely the UNIFLEX-PM application, on the basis of its stochastic behaviour. Taking into account this indicator, possible impacts deriving from the adoption of the UNIFLEX-PM system have been "measured" through a quantitative comparison between such a solution and an $\mathrm{M}^{2} \mathrm{LC}$ based HVDC application in the back to back configuration, selected as reference case. This architecture has been selected because it has functionalities similar to the ones of the UNIFLEX-PM system and has been already utilised for building some commercial products.

When repairable systems are analysed, the estimate of their availability may provide fair information to judge the real effectiveness of the adopted solution. For such reasons, a detailed availability study has been performed for the two systems through a dedicated software tool, based on the Monte Carlo method and developed in the Matlab ${ }_{\circledast} 7.0$ environment. Simulations have been carried out considering three different operating conditions and, for the UNIFLEXPM system, two technologies for the capacitors (electrolytic or film) and two redundancy strategies.

The results have shown that over an operating time horizon of 90000 hours the two systems have similar performances in terms of availability: around 99.1\% for UNIFLEX-PM and around 99.8\% for the reference case (no redundancy and electrolytic capacitors for the former). On the contrary, the reference case has definitely better performances in terms of number of failures: about 3.5 compared with 29 for the basic architecture of UNIFLEX-PM. The results have also shown that such advantage for the reference case is only apparent because it is possible for UNIFLEX-PM to achieve similar performances, both in terms of availability and number of failures, if a redundancy strategy or film capacitors for the DC link are used.

At last, a study was also dedicated to the analysis of the impacts deriving form the adoption of preventive maintenance on the electrolytic capacitors aimed at avoiding failures due to wear-out. The results of such study have shown that preventive maintenance actions should be duly planned in order to exploit the achievable benefits, in terms of reduction of the number of failures and increase of the availability performances, obviously paying particular attention to the economic aspects of the adopted solution. If, on one hand, a suitable value of the frequency and speed of the preventive actions is mandatory to optimise the previously mentioned benefits, on the other hand, the choice of those parameters could have a not negligible impact on operation and support costs.

To conclude, the dependability prediction testifies the proposed modular multilevel architecture has all the potentialities for a future industrialisation, being the stochastic performances of the UNIFLEX-PM application fully comparable with the ones provided by similar architectures already used for producing commercial products.

\section{Acknowledgment}

The activities presented in the paper have been developed in the framework of the European Project "Advanced power converters for universal and flexible power management in future electricity network", EC Contract no. 019794 (SES6). The authors would like to thank all the Partners of the UNIFLEX-PM project Consortium for their support.

\section{References}

[1] EUR 20901, "New ERA for electricity in Europe", European Commission Directorate-General for Research, Office for Official Publications of the European Communities, Luxembourg, ISBN 92-894-6262-0.

[2] F. Iov, F. Blaabjerg, R. Bassett, J. Clare, A. Rufer, S. Savio, P. Biller, P. Taylor, B. Sneyers "Advanced Power Converter for Universal and Flexible Power Management in Future Electricity Network”, CIRED 2007 Conference, May 2007, Wien, Austria.

[3] A. Watson, H.Q.S. Dang, J. Clare, P. Wheeler, G. Mondal, S. Kenzelmann, Y.R. de Novaes, A. Rufer, "A Novel Multilevel Converter Structure Integrated into Power Systems and its Performance Evaluation”, EPE 2009 Conference, September 2009, Barcelona, Spain.

[4] F. Schettler, H. Huang, N. Christl, "HVDC Transmission System using Voltage Sourced Converters - Design and Applications”, IEEE Power Engineering Society Summer Meeting, Seattle, July 2000.

[5] A. Lesnicar, R. Marquardt, "An innovative modular multilevel converter topology suitable for a wide power range”, IEEE PowerTech Conference 2003, Bologna, Italy, June 2003.

[6] M. Glinka, R. Marquardt, "A new AC/AC multilevel converter family", IEEE Transactions on Industrial Electronics, Vol. 52, No. 3, June 2005.

[7] S. Allebrod, R. Hamerski, R. Marquardt, "New transformerless, scalable modular multilevel converters for HVDC transmission”, IEEE Power Electronic Specialists Conference 2008, Rhodes, Greece, June 2008.

[8] S. Allebrod, R. Hamerski, R. Marquardt, "New transformerless, scalable modular multilevel converters for HVDC transmission”, IEEE Power Electronic Specialists Conference 2008, June 2008, Rhodes, Greece.

[9] IEC EN 61709, "Electronic components - Reliability Reference conditions for failure rates and stress models for conversion”, March 1996.

[10] IEC TR 62380, "Reliability data handbook: universal model for reliability prediction of electronics components, PCBs and equipment”, August 2004.

[11] IEEE Gold book, "IEEE recommended practice for the design of reliable industrial and commercial power systems”, August 1998, ISBN-1-55937-969-3.

[12] US MIL-HDBK-217F, "Reliability prediction for electronic equipment”, December 1991, + Notice 1, July 1992, + Notice 2, February 1995.

[13] K. C. Kapur, R. L. Lamberson, "Reliability in Engineering Design”, John Wiley and Sons, Chichester, 1977. 